\title{
Pseudo Leja sequences
}

\section{Leokadia Białas-Cież • Jean-Paul Calvi}

Received: 17 June 2010 / Accepted: 27 October 2010 / Published online: 17 November 2010

(C) The Author(s) 2010. This article is published with open access at Springerlink.com

\begin{abstract}
We study pseudo Leja sequences attached to a compact set in the complex plane. The requirements are weaker than those of ordinary Leja sequences, but these sequences still provide excellent points for interpolation of analytic functions and their computation is much easier. We also apply them to the construction of excellent sets of nodes for multivariate interpolation of analytic functions on product sets.
\end{abstract}

Keywords Leja sequences · Equilibrium measure - Lagrange interpolation · Markov inequality $\cdot$ Alper smooth curves $\cdot($ Weakly) admissible meshes

Mathematics Subject Classification (2000) $\quad 41 \mathrm{~A} 05 \cdot 41 \mathrm{~A} 63 \cdot 65 \mathrm{E} 05$

\section{Introduction}

Let $K$ be a nonempty compact subset of the complex plane, and let $\left(a_{n}\right)$ be a sequence of points in $K$. One says that $\left(a_{n}\right)$ is a Leja sequence for $K$ if the following extremal metric property holds true,

$$
\begin{gathered}
\left|w_{n}\left(a_{n}\right)\right|=\max _{z \in K}\left|w_{n}(z)\right|, \quad n \geq 1, \quad \text { where } \\
w_{n}=\left(\cdot-a_{0}\right) \cdots\left(\cdot-a_{n-1}\right), \quad n \geq 1 .
\end{gathered}
$$

Thus, the $(n+1)$-st term $a_{n}$ of a Leja sequence must maximize the product of the distances to the $n$ previous ones. Such sequences were first considered by Albert Edrei to obtain a new

L. Białas-Cież $(\bowtie)$

Institute of Mathematics, Jagiellonian University,

Łojasiewicza 6, 30-348 Kraków, Poland

e-mail: Leokadia.Bialas-Ciez@im.uj.edu.pl

J.-P. Calvi

Institut de Mathématiques, Université de Toulouse III and CNRS (UMR 5219),

31062 Toulouse Cedex 9, France

e-mail: jpcmath@netscape.net 
way of computing the transfinite diameter of a compact set [13, pp. 78-79 and lemma 10]. The current interest in these sequences, however, originated from a later independent work of Franciszek Leja [18] who used them to reconstruct Green functions of (the complement in $\overline{\mathbb{C}}$ of) regular polynomially convex compact sets. By regular compact set, we mean a nonpolar (i.e. of positive logarithmic capacity) compact set for which the Green function $G_{K}$ extends to a continuous function on the whole plane. Recall that $K$ is polynomially convex if $K=\left\{z:|p(z)| \leq\|p\|_{K}\right.$ for all polynomials $\left.p\right\}$.

With the above assumptions on $K$, Leja sequences furnish excellent points for polynomial interpolation of analytic functions. Namely, the Lagrange interpolation polynomial $\mathbf{L}\left[a_{0}, \ldots, a_{n} ; f\right]$ at the $n+1$ nodes $a_{0}, \ldots, a_{n}$, of a function $f$ analytic in a neighborhood of $K$-we write $f \in \mathscr{A}(K)$ — converges uniformly (and geometrically fast) to $f$ on $K$ as $n$ goes to $\infty$. A sequence of interpolation points satisfying such a property is said to be extremal for $K$. Using the classical notation for divided differences, the Newton series expansion of $f$,

$$
f=\sum_{n=0}^{\infty} f\left[a_{0}, \ldots, a_{n}\right] w_{n},
$$

actually converges on every compact set

$$
K_{r}:=\left\{G_{K} \leq \log r\right\},
$$

to which $f$ extends analytically. In particular, Newton's basis $\left(w_{n}\right)$ associated with an extremal sequence forms a topological basis of the space $\mathscr{A}(K)$ endowed with its standard topology.

Fekete points are other well-known excellent points for polynomial interpolation. They are defined in a similar fashion. A subset of $n+1$ points $b_{0}^{n}, \ldots, b_{n}^{n}$ in $K$ is said to form a Fekete set of order $n$ if its points maximize the product of their mutual distances on $K$, that is,

$$
\left|\operatorname{VDM}\left(b_{0}^{n}, \ldots, b_{n}^{n}\right)\right|=\max \left\{\left|\operatorname{VDM}\left(z_{0}, \ldots, z_{n}\right)\right|,\left(z_{0}, \ldots, z_{n}\right) \in K^{n+1}\right\},
$$

where VDM denotes the vandermondian of the $z_{i}$ 's,

$$
\operatorname{VDM}\left(z_{0}, \ldots, z_{n}\right)=\prod_{0 \leq i<j \leq n}\left(z_{i}-z_{j}\right) .
$$

The main difference between Leja and Fekete points is that the latter form an array rather than a sequence. When we go from $n$ to $n+1$, we get $n+2$ new Fekete points whereas we get only one new Leja point. Working with sequences rather than with arrays has two obvious advantages. From a theoretical point of view, sequences give rise to polynomial bases of $\mathscr{A}(K)$, and from a computational point of view, we may use calculations made to get $\mathbf{L}\left[a_{0}, \ldots, a_{n} ; f\right]$ in the case where we need to compute $\mathbf{L}\left[a_{0}, \ldots, a_{n+k} ; f\right]$. Our main interest in Leja sequences here came from a third, less known advantage: they enable one to construct extremal sets of interpolation points for multivariate Lagrange interpolation on cartesian products of planar sets. We shall explain this in details in Sect. 5.

Unfortunately, although it is readily seen that every compact set (containing at least two points) admits infinitely many Leja sequences, it is in general impossible to compute Leja (or Fekete) points, except for a very small value of $n$. Explicit expressions of the $n$-th element of a Leja sequence seem to be currently available only in the case of disks, see Sect. 3.

In this paper, we study a class of sequences obtained by weakening the definition of Leja sequences. We shall no longer look for a point $a_{n}$ satisfying $\left|w_{n}\left(a_{n}\right)\right|=\max _{z \in K}\left|w_{n}(z)\right|$ as 
in (1) but rather

$$
M_{n}\left|w_{n}\left(a_{n}\right)\right| \geq \max _{z \in K}\left|w_{n}(z)\right|,
$$

where $\left(M_{n}\right)$ is a sequence of moderate growth, and in the best case, a positive constant greater than 1, see Definition 1. We shall see that such pseudo Leja sequences still provide extremal sequences for polynomial interpolation, and at the same time, interesting information can be obtained, including explicit examples, without much difficulty. In contrast with the classical case, such sequences can be rather easily computed. Our algorithm enables one to compute the first $n$ points of a pseudo Leja sequence for many reasonable (finitely connected) compact sets. We use a natural discretization process which, as far as we know, goes back to Saff and Totik [26, Section 5.1]. The main difference here lies in our use of the notion of admissible meshes and weakly admissible meshes recently introduced in [10]. Many of our results readily extend to the weighted case as long as we work with compact sets and bounded weights. The application to multivariate Lagrange interpolation is given in the last section. We tried to provide there enough details to be accessible to nonspecialists in multivariate polynomial approximation.

For results on Leja sequences and various fields of applications, we refer to [3,12,25], and the references therein. Of particular interest is [3] where the authors constructed efficient sequences that they called fast Leja sequences (mainly in the case of an interval) by selecting points over carefully chosen sets of interlacing points. It would be interesting to know whether such fast Leja sequences are examples of our pseudo Leja sequences.

We shall assume that the reader is familiar with the basic notions of potential theory. An excellent reference is the book of Ransford [24]. We shall mainly use three objects attached to a compact subset $K$ : the Green function $G_{K}$ that we already mentioned, the logarithmic capacity $C(K)$ and the various ways of obtaining it (as the transfinite diameter, the Chebyshev constant, or the conformal radius of $K$ ), and the equilibrium measure $\mu_{K}$.

Notation. $D(z, r)$ denotes the closed disk of center $z$ and radius $r$. The open disk is denoted by Int $D(z, r) ; C(z, r)$ is the circle of center $z$ and radius $r$.

\section{Pseudo Leja sequences}

\subsection{Definition}

We assume that $K$ is a polynomially convex compact subset of $\mathbb{C}$. Recall that a sequence of real numbers $\left(M_{n}\right)$ is said to be of subexponential growth, if

$$
\lim _{n \rightarrow \infty} M_{n}^{1 / n}=1 .
$$

Some say polynomial growth instead of subexponential growth.

Definition 1 Let $\left(a_{n}\right)$ be a sequence of points in $K$ and $\left(M_{n}\right)$ a sequence of subexponential growth with $M_{n} \geq 1, n \in \mathbb{N}$. We say that $\left(a_{n}\right)$ is a pseudo Leja sequence of Edrei growth $M_{n}$ if

$$
M_{n}\left|w_{n}\left(a_{n}\right)\right| \geq \max _{z \in K}\left|w_{n}(z)\right|, \quad w_{n}=\left(\cdot-a_{0}\right) \cdots\left(\cdot-a_{n-1}\right), \quad n \in \mathbb{N}^{\star} .
$$

No condition is set on the first point (apart from being an element of $K$ ). Every pseudo Leja sequence of Edrei growth $M_{n}$ is also of Edrei growth $M_{n}^{\prime}$ whenever $M_{n} \leq M_{n}^{\prime}$. An ordinary Leja sequence is a pseudo Leja sequence of Edrei growth 1. In view of the 
maximum principle, the elements of genuine Leja sequences necessarily lie on the boundary of $K$. This is no longer true in the case of pseudo Leja sequences. The points need not be on the boundary and it is even readily seen that if $K$ is a compact set of nonempty interior $\operatorname{int}(K)$ such that $K=\overline{\operatorname{int}(K)}$, then we may construct pseudo Leja sequences with no point at all on the boundary.

It is also worth noting that pseudo Leja sequences are invariant under an affine map $L: z \rightarrow a z+b$, that is, $\left(a_{n}\right)$ is a pseudo sequence of Edrei order $M_{n}$ for $K$ if and only if $\left(L\left(a_{n}\right)\right)$ is a pseudo Leja sequence of the same Edrei growth for $L(K)$.

\subsection{Asymptotic behavior}

The following theorem gives the property of pseudo Leja sequences most important to us. The proof requires only a slight adaptation of the classical one.

Theorem 1 Let $K$ be a nonpolar, polynomially convex, compact set in the plane. If $\left(a_{n}\right)$ is a pseudo Leja sequence of any Edrei growth and if $\mu_{n}$ denotes the probability measure obtained by assigning a mass of $1 /(n+1)$ to every $a_{i}$ with $0 \leq i \leq n$, that is,

$$
\mu_{n}=\frac{1}{n+1} \sum_{i=0}^{n}\left[a_{i}\right],
$$

where $\left[a_{i}\right]$ denotes the Dirac measure on $a_{i}$, then the sequence $\left(\mu_{n}\right)$ converges weak- $\star$ to the equilibrium measure $\mu_{K}$ of $K$,

$$
\mu_{n} \stackrel{\star}{\longrightarrow} \mu_{K}, \quad n \rightarrow \infty
$$

Observe that any assumption of the regularity or of the growth of $M_{n}$ (apart from Eq. 8) is not necessary in the above theorem.

This result has two classical consequences.

1. The sequence $(1 / n) \log \left|w_{n}\right|, n \in \mathbb{N}^{\star}$ converges to $G_{K}+\log C(K)$ locally uniformly on $\mathbb{C} \backslash K$.

2. $\left(a_{n}\right)$ is an extremal sequence for Lagrange interpolation of analytic function on $K$.

It is well known that the fact that Theorem 1 implies (1) is an easy consequence of Riesz's representation of Green functions. Details can be found e.g. in [6]. That (1) implies (2) is the classical Kalmar-Walsh theorem (see $[31,28,16])$. Observe that the weak convergence of $\mu_{n}$ to the equilibium measure is a necessary condition for $\left(a_{n}\right)$ to be extremal only in the case where the points are required to lie on the boundary of $K$. In the general case, the necessary condition is more complicated; the balayage on $\partial K$ of the limit of any converging subsequence of $\mu_{n}$ must be equal to the equilibrium measure, see [7, corollary 1]. The balayage on $\partial K$ of a positive measure $\mu$ supported on $K$ is the unique positive measure $b(\mu)$ supported on $\partial K$ such that $\int f d \mu=\int f d b(\mu)$ for every function $f$ continuous on $K$ and harmonic on the interior of $K$, see [7, proposition 1].

Now, Theorem 1 is a consequence of the following proposition.

Theorem 2 Under the same assumptions as in Theorem 1, we have

$$
\lim _{n \rightarrow \infty}\left|\operatorname{VDM}\left(a_{0}, \ldots, a_{n}\right)\right|^{\frac{2}{n(n+1)}}=C(K),
$$

where $C(K)$ stands for the logarithmic capacity of $K$. 
Proof To prove (12), we first observe that if $\left(b_{0}^{n}, \ldots, b_{n}^{n}\right)$ is a set of Fekete points of order $n \in \mathbb{N}$, then $\left|\operatorname{VDM}\left(a_{0}, \ldots, a_{n}\right)\right| \leq\left|\operatorname{VDM}\left(b_{0}^{n}, \ldots, b_{n}^{n}\right)\right|$ and hence

$$
\limsup _{n \rightarrow \infty}\left|\operatorname{VDM}\left(a_{0}, \ldots, a_{n}\right)\right|^{\frac{2}{n(n+1)}} \leq \lim _{n \rightarrow \infty}\left|\operatorname{VDM}\left(b_{0}^{n}, \ldots, b_{n}^{n}\right)\right|^{\frac{2}{n(n+1)}}=C(K) .
$$

On the other hand, we have

$$
\left.\operatorname{VDM}\left(a_{0}, \ldots, a_{n}\right)\right|^{\frac{2}{n(n+1)}}=\left\{\prod_{k=1}^{n} \prod_{l=0}^{k-1}\left|a_{k}-a_{l}\right|\right\}^{\frac{2}{n(n+1)}}=\left\{\prod_{k=1}^{n}\left|w_{k}\left(a_{k}\right)\right|\right\}^{\frac{2}{n(n+1)}}
$$

Since, by the definition of a pseudo Leja sequence of Edrei growth $M_{n}$

$$
\left|w_{k}\left(a_{k}\right)\right| \geq\left(1 / M_{k}\right) \max _{z \in K}\left|w_{k}\right|, \quad \text { and } \max _{z \in K}\left|w_{k}\right| \geq m_{k}(K),
$$

where $m_{k}(K)$ is the lower bound of $\max _{z \in K}|p|$ when $p$ runs among the monic polynomial of degree $k$, it follows that

$$
\left.\operatorname{VDM}\left(a_{0}, \ldots, a_{n}\right)\right|^{\frac{2}{n(n+1)}} \geq\left(\prod_{k=1}^{n} \frac{m_{k}(K)}{M_{k}}\right)^{\frac{2}{n(n+1)}} .
$$

Since $m_{n}(K)^{1 / n} \rightarrow C(K)$ (this is the characterization of logarithmic capacity in terms of the Chebyshev constant) and $M_{n}^{1 / n} \rightarrow 1$, we have

$$
\left\{\frac{m_{n}(K)}{M_{n}}\right\}^{1 / n} \rightarrow C(K), \quad n \rightarrow \infty
$$

from which we readily deduce that

$$
\left(\prod_{k=1}^{n} \frac{m_{k}(K)}{M_{k}}\right)^{\frac{2}{n(n+1)}} \rightarrow C(K), \quad n \rightarrow \infty .
$$

Hence,

$$
\liminf _{n \rightarrow \infty}\left|\operatorname{VDM}\left(a_{0}, \ldots, a_{n}\right)\right|^{\frac{2}{n(n+1)}} \geq C(K),
$$

and this achieves the proof of (12).

The fact that Theorem 2 implies Theorem 1 is shown in [6, Theorem 1.5]. There is a small inaccuracy in the proof given in this reference. Contrary to what is said there, the measure $\tilde{\mu}_{d}$ used in $\left[6,1.7\right.$, p. 447] is not, in general, a probability measure because the sets $\Delta_{d j}$ are not necessarily pairwise disjoint. The correct definition for the sequence $\tilde{\mu}_{d}$ is the following. Given $z_{d j}, j=0, \ldots, d$ as in $\left[6,1.7\right.$, p. 447], we choose $r_{d}<1 / \sqrt{d}$ small enough to have $\left|z_{d j}-z_{d i}\right|>r_{d}$ for $i \neq j$. We then define $\Delta_{d j}$ as the disk of center $z_{d j}$ and radius $r_{d}, \tilde{\mu}_{d}:=\frac{1}{d+1} \sum_{j=0}^{d} d m_{j}$ where $d m_{j}$ is the Lebesgue measure on $\Delta_{d j}$ normalized so that its mass on $\Delta_{d j}$ equals 1 . 


\subsection{Remarks on Theorem 1}

(A) Although (all) the points of a pseudo Leja sequence may fail to lie on the boundary of $K$, the number of points at a positive distance from the boundary is actually small in the sense that if $S$ is a compact subset in the interior of $K$ then

$$
\frac{1}{n+1} \sharp\left(S \cap\left\{a_{0}, \ldots, a_{n}\right\}\right) \rightarrow 0, \quad n \rightarrow \infty .
$$

(B) A pseudo Leja sequence is necessarily very chaotic. Since the support of $\mu_{K}$ is the boundary of $K$, every point there must be a limit point of $\left(a_{n}\right)$. Phung Van Manh [19] showed us an example of pseudo Leja sequence of increasing Edrei growth for the unit disk with a limit point at the origin. We do not know whether the same phenomenon may occur in the case of pseudo Leja sequences of constant Edrei growth.

(C) Relation (11) is weaker than the property of being a pseudo Leja sequence. Indeed, it is easy to find a sequence satisfying (11) without being a pseudo Leja sequence. Here is a way of constructing such a sequence. Let $\left(a_{n}\right)$ be an ordinary Leja sequence for $K$. We inductively define a sequence $\left(b_{n}\right)$ as follows: $b_{0}=a_{0}$, and for $n \geq 0, b_{n+1}=$ $a_{n+1}$ if $n+1$ is not a square, otherwise $b_{n+1}$ is chosen close enough to $b_{n}$ to have $(n+1)^{n+1} w_{n+1}\left(b_{n+1}\right)<\max _{z \in K}\left|w_{n+1}(z)\right|$. The sequence $\mu_{n}=\frac{1}{n+1} \sum_{i=0}^{n}\left[b_{i}\right]$ has the same asymptotic behavior as $\mu_{n}=\frac{1}{n+1} \sum_{i=0}^{n}\left[a_{i}\right]$ because the $\left[b_{i}\right]$ 's defer from the $\left[a_{i}\right]$ for at most $\sqrt{n}$ values of $i$. But the definition of $\left(b_{n}\right)$ ensures that it is not a pseudo Leja sequence.

(D) One might be surprised that the relatively weak assumption on $M_{n}$ enables one to recover the equilibrium measure. Theorem 1, however, does not give quantitative results. For efficient practical computations, we must have good results for a relatively small $n$. This cannot be achieved if the numbers $M_{n}$ are very big for small $n$, simply because, in this case, the conditions on the first points $a_{n}$ are very weak. Apart from that, numerical evidence suggests that slowly growing $M_{n}$ provides a rapid convergence to the equilibrium measure. We may also notice that, in Theorem 4 below, the growth of $M_{n}$ has a very limited influence on the estimate. In any case, in practice, we shall only look for pseudo Leja sequences of constant or slowly increasing Edrei growth.

(E) The property of being an extremal sequence does not imply a good behavior of the Lebesgue constant (see 4.2 for a Definition). Thus, there is a strong difference between the interpolation of analytic functions and the interpolation of differentiable functions. Taylor and Totik [29] recently showed that genuine Leja sequences of many plane compact sets have a Lebesgue constant of subexponential growth, thus answering positively a question posed many years ago in [6]. It seems that the argument given by Taylor and Totik works as well without change in the case of our pseudo Leja sequences of constant growth $\left(M_{n}=M>1\right)$ but we shall not discuss this question further here.

\subsection{On the distribution of pseudo Leja sequences}

We give two elementary estimates on the distribution of the points of a pseudo Leja sequence. Both of them rely on the Markov inequality satisfied by the compact set, and they suggest that the sequence $\left(M_{n}\right)$ has a relatively limited influence on the distribution of the points.

We say that a (polynomially convex) compact set $K$ is a $(\delta, r)$ compact set if the following Markov inequality holds true

$$
\max _{z \in K}\left|p^{\prime}(z)\right| \leq \delta(\operatorname{deg} p)^{r} \max _{z \in K}|p(z)|
$$


for all polynomials $p$. The number $r$ is the exponent and the number $\delta$ the constant of the Markov inequality (13). A compact set satisfying such an inequality is not polar, see [4]. A universal Markov inequality for connected compact sets is recalled in the following subsection 4.3. From (13), one readily deduces a bound for the other derivatives,

$$
\max _{z \in K}\left|p^{(j)}(z)\right| \leq \delta^{j}(d(d-1) \cdots(d-j+1))^{r} \max _{z \in K}|p(z)|
$$

Theorem 3 Let $K$ be a $(\delta, r)$-compact subset of $\mathbb{C}$ and $\left(a_{n}\right)$ a pseudo Leja sequence of Edrei growth $\left(M_{n}\right)$ for $K$. We have

$$
\left|a_{d}-a_{j}\right| \geq \log \left(1+1 / M_{d}\right) /\left(\delta d^{r}\right), \quad j=0, \ldots, d-1 .
$$

Proof Fix $j \in\{0, \ldots, d-1\}$. Starting from the definition of $a_{d}$, we have

$$
\begin{aligned}
\max _{z \in K}\left|w_{d}(z)\right| & \leq M_{d}\left|w_{d}\left(a_{d}\right)\right|=M_{d}\left|w_{d}\left(a_{d}\right)-w_{d}\left(a_{j}\right)\right| \\
& \leq M_{d} \sum_{l=1}^{d}\left|w_{d}^{(l)}\left(a_{j}\right)\right|\left|a_{d}-a_{j}\right|^{l} / l !
\end{aligned}
$$

Now, using Markov's inequality (14) to estimate $\left|w_{d}^{(l)}\left(a_{j}\right)\right|$, we arrive at

$$
\begin{aligned}
\max _{z \in K}\left|w_{d}(z)\right| & \leq M_{d} \max _{z \in K}\left|w_{d}(z)\right| \sum_{l=1}^{d} \delta^{l}(d(d-1) \cdots(d-l+1))^{r} \frac{\left|a_{d}-a_{j}\right|^{l}}{l !} \\
& \leq M_{d} \max _{z \in K}\left|w_{d}(z)\right|\left(\exp \left(\delta d^{r}\left|a_{d}-a_{j}\right|\right)-1\right) .
\end{aligned}
$$

It follows that $M_{d}\left(\exp \left(\delta d^{r}\left|a_{d}-a_{j}\right|\right)-1\right) \geq 1$ which is the required estimate.

When $K$ is a $(\delta, r)$ convex set, using the mean value theorem instead of a Taylor expansion, we obtain a slightly more precise inequality. Namely,

$$
\left|a_{d}-a_{j}\right| \geq 1 /\left(\delta M_{d} d^{r}\right), \quad j=0, \ldots, d-1 .
$$

Clearly, having constructed the first $d$ points of a pseudo Leja sequence of Edrei growth $\left(M_{n}\right)$, there are many candidates for $a_{d}$. The following observation gives a measure of the set of all possible $a_{d}$. It would also provide another way to arrive at one of the algorithms given later.

Theorem 4 Under the assumptions of Theorem 3 , there exists a closed disk $D$ of radius $\log \left(2-1 / M_{d}\right) /\left(\delta d^{r}\right)$ such that $D \cap K \neq \emptyset$ and every element a of $D$ satisfies $M_{d}\left|w_{d}(a)\right| \geq$ $\max _{z \in K}\left|w_{d}(z)\right|$.

Proof Let $z_{0} \in \partial K$ such that

$$
\left|w_{d}\left(z_{0}\right)\right|=\max _{z \in K}\left|w_{d}(z)\right|
$$

and let

$$
z \in D\left(z_{0}, \mu /\left(\delta d^{r}\right)\right) \quad \text { with } \mu=\log \left(2-1 / M_{d}\right)
$$


We want to prove that $\left|w_{d}\left(z_{0}\right)\right| \leq M_{d}\left|w_{d}(z)\right|$. Using again the iterated Markov inequality (14) and the Taylor expansion of $w_{d}$ at $z_{0}$, we obtain

$$
\begin{aligned}
|| w_{d}(z) \mid & -\left|w_{d}\left(z_{0}\right)\right||\leq| w_{d}(z)-w_{d}\left(z_{0}\right)\left|\leq \sum_{k=1}^{d}\right| w_{d}^{(k)}\left(z_{0}\right) \mid \frac{\left|z-z_{0}\right|^{k}}{k !} \\
& \leq \max _{z \in K}\left|w_{d}(z)\right| \cdot \sum_{k=1}^{d} \frac{\mu^{k}}{k !} \leq\left(e^{\mu}-1\right) \max _{z \in K}\left|w_{d}(z)\right| .
\end{aligned}
$$

Since $\left|w_{d}\left(z_{0}\right)\right|=\max _{z \in K}\left|w_{d}(z)\right|$, it follows that

$$
\max _{z \in K}\left|w_{d}(z)\right|\left(2-e^{\mu}\right) \leq\left|w_{d}(z)\right| .
$$

Since $\left(2-e^{\mu}\right)=1 / M_{d}$, the estimate is established.

\section{Explicit pseudo Leja sequences}

\subsection{Leja sequences on disks}

In [3], the authors mention without proof that if $K=D(0,1)$ then a Leja sequence for which $e_{0}=1$ is given by

$$
e_{n}=\exp \left(i \pi \sum_{k=0}^{s} j_{k} 2^{-k}\right) \text { for } n=\sum_{k=0}^{s} j_{k} 2^{k}, \quad j_{k} \in\{0,1\} .
$$

In particular, the $2^{s}$ first points form a complete set of roots of unity of degree $2^{s}$. There are probably few, if any, other classes of compact sets for which explicit computable expressions can be expected. For the convenience of the reader, we give a proof of this result. We actually give a description of all the Leja sequences of the disk. In view of the invariance under affine maps recalled in Sect. 2.1, we may limit ourselves to the sequences of the unit disk satisfying $a_{0}=1$.

The $k$-th section of $\left(a_{n}\right)$ is the finite sequence $a^{k}:=\left(a_{0}, \ldots, a_{k-1}\right)$. Given $\left(a_{n}\right)$ and $\left(b_{n}\right)$ we denote by $\left(a^{s}, b^{q}\right)$ the finite sequence $\left(a_{0}, \ldots, a_{s-1}, b_{0}, \ldots, b_{q-1}\right)$.

Theorem 5 The structure of a Leja sequence $\left(a_{n}\right)$ of the unit disk with $a_{0}=1$ is given by the following rule. The underlying set of the $2^{n}$-th section consists of the $2^{n}$-th roots of the unity, and if the $2^{n}$-th section is known, then the $2^{n+1}$ section is

$$
\left(a^{2^{n}}, \rho b^{2^{n}}\right),
$$

where $\rho$ is any solution of $z^{2^{n}}=-1$ and $b^{2^{n}}$ is the section of a Leja sequence for the unit disk with $b_{0}=1$.

Proof The proof is by double induction. The result is obviously true when $n=0$ for the 2 -nd section of a Leja sequence with $a_{0}=1$ is $(1,-1)$. We assume that $\left\{a_{k}: k<2^{n}\right\}$ is the set of the $2^{n}$-th roots of unity, and we prove that $a^{2^{n+1}}$ is as claimed in (24) which, in turn, readily implies that the underlying set of the $2^{n+1}$-st section consists of the $2^{n+1}$-st roots of unity. This is the induction step on $n$. Thus, setting $m=2^{n}$, we must show that

$$
a_{m+k}=\rho b_{k}, \quad k=0, \ldots, 2^{n}-1 .
$$

The proof of this claim is by induction on $k$. 
A) The case $k=0$. We look for a point $a_{m}$ on the unit disk that maximizes

$$
\left|w_{m}(z)\right|=\prod_{a \in a^{2^{n}}}|z-a|=\left|z^{2^{n}}-1\right|, \quad|z|=1,
$$

where the second equality comes from the induction hypothesis on $n$. Thus, any of the $2^{n}$ roots of the equation $z^{2^{n}}=-1$ can be chosen as $a_{m}$. Let us call $\rho$ the particular root we choose so that $a_{m}=\rho=\rho b_{0}$ with $b_{0}=1$. This proves (25) in the case $k=0$.

B) Let now $0 \leq k<2^{n}-1$. We assume that $a_{m+j}=\rho b_{j}$ for $j=0, \ldots$, $k$ where the $(k+1)$-tuple $\left(b_{0}, b_{1}, \ldots, b_{k}\right)$ is the $(k+1)$-st section of a Leja sequence for the unit disk with $b_{0}=1$ and prove that if $b_{k+1}$ is defined by $a_{m+k+1}=\rho b_{k+1}$, then

$$
\prod_{j=0}^{k}\left|b_{k+1}-b_{j}\right|=\max _{|z|=1} \prod_{j=0}^{k}\left|z-b_{j}\right| \text {. }
$$

Let us write

$$
W_{k}(z)=\left(z-b_{0}\right) \cdots\left(z-b_{k}\right) .
$$

To prove our claim, we just need to check that the complex number $b_{k+1}$ defined above does satisfy

$$
\left|W_{k}\left(b_{b+1}\right)\right|=\max _{|z|=1}\left|W_{k}(z)\right| .
$$

By definition, $a_{m+k+1}$ must be chosen in order to maximize the following quantity on the unit circle,

$$
\left|w_{m+k}(z)\right|=\left|z-a_{0}\right| \cdots\left|z-a_{2^{n}-1}\right|\left|z-a_{m}\right|\left|z-a_{m+1}\right| \cdots\left|z-a_{m+k}\right| .
$$

However, by the induction hypothesis on $n$, the numbers $a_{0}, \ldots, a_{m}$ form a complete set of $2^{n}$-th roots of unity. Thus,

$$
\left(z-a_{0}\right) \cdots\left(z-a_{2^{n}-1}\right)=z^{2^{n}}-1 .
$$

On the other hand, by the assumption hypothesis on $k$, we have $a_{m+j}=\rho b_{j}, 0 \leq j \leq k$. Thus, Eq. (28) reduces to

$$
\left|w_{m+k}(z)\right|=\left|z^{2^{n}}-1\right|\left|z-\rho b_{0}\right|\left|z-\rho b_{1}\right| \cdots\left|z-\rho b_{k}\right| .
$$

Now, observe that the rotation $z \rightarrow \rho z$ leaves the unit disk invariant. This yields

$$
\begin{aligned}
\max _{|z|=1}\left|w_{m+k}(z)\right| & =\max _{|z|=1}\left|w_{m+k}(\rho z)\right| \\
& =\max _{|z|=1}\left|(\rho z)^{2^{n}}-1\right|\left|\rho z-\rho b_{0}\right|\left|\rho z-\rho b_{1}\right| \cdots\left|\rho z-\rho b_{k}\right| \\
& =\max _{|z|=1}\left\{\left|W_{k}(z)\right| \cdot\left|z^{2^{n}}+1\right|\right\}
\end{aligned}
$$

where we used the fact that $\rho^{2^{n}}=-1$ and the definition of $W_{k}$ given in (27). Here, both factors on the right-hand side attain their maximum on the subset $\left\{z^{2^{n}}=1\right\}$, and on this subset, the second factor is constant. Hence,

$$
\max _{|z|=1}\left|w_{m+k}(z)\right|=2 \max _{|z|=1}\left|W_{k}(z)\right| .
$$


To say that $\left|W_{k}\right|$ attains its maximum on $\left\{z^{2^{n}}=1\right\}$, we needed the fact that, by induction hypothesis, the $b_{i}$ 's are Leja points of rank not greater than $2^{n}-1$. The computation described previously shows that if $a_{m+k+1}$ maximizes $\max \left|w_{m+k}(z)\right|$, then $b_{k+1}=a_{m+k+1} / \rho$ maximizes $\max _{|z|=1}\left|W_{k}(z)\right|$ as was to be proved.

Corollary 1 Let $N_{n}$ denote the number of $2^{n}$-th sections of Leja sequences for the unit disk satisfying $a_{0}=1$. We have

$$
\left(\log N_{n}\right)^{1 / n} \rightarrow 2, \quad n \rightarrow \infty .
$$

Proof Let $L_{n}:=\log N_{n}$. We deduce from (24) that $N_{1}=1$ and $N_{n+1}=2^{n} N_{n}^{2}$, hence $L_{1}=0$ and $L_{n+1}=2 L_{n}+n \log 2, n \geq 1$. This relation implies that if $f(z)=\sum_{n=1}^{\infty} L_{n} z^{n-1}$, then $f(z)=2 z f(z)+z \log 2 /(1-z)^{2}$. The smallest singularity of $f$ is $1 / 2$, and the result follows.

A more precise analysis would give an asymptotic formula for $N_{n}$. Here, we shall only observe that the number obtained is quite big and it would be interesting to study whether some Leja sequences have computational advantages over others.

Corollary 2 A particular Leja sequence is given by $a_{0}=1, a_{1}=-1$, and

$$
a^{2^{n+1}}=\left(a^{2^{n}}, e^{\frac{i \pi}{2^{n}}} a^{2^{n}}\right), \quad n \geq 1 .
$$

This Leja sequence is the sequence $\left(e_{n}\right)$ defined in (23).

Proof We sketch a proof of the second part. We show that if $m=2^{n}+t$ with $t \in\{1, \ldots$, $\left.2^{n}-1\right\}$, then $e_{m}=e^{i \pi / 2^{n}} e_{t}$. Let $t=\sum_{k=0}^{n-1} j_{k} 2^{k}$, then

$$
\exp \left(i \pi / 2^{n}\right) e_{t}=\exp \left(i \pi / 2^{n}\right) \exp \left(i \pi \sum_{k=0}^{n-1} j_{k} 2^{-k}\right)=\exp \left(i \pi \sum_{k=0}^{n-1} j_{k} 2^{-k}+2^{-n}\right)=e_{m} .
$$

Corollary 3 Let $K$ be a nonpolar polynomially convex compact subset of the plane. Let $\phi$ be the conformal mapping of the exterior of the unit disk to the exterior of $K$. If $\phi$ extends to a homeomorphism from the unit $\{|z|=1\}$ onto the boundary of $K$ then, for every Leja sequence $\left(a_{n}\right)$ of the unit disk, $\phi\left(a_{n}\right)$ is an extremal sequence for $K$.

Proof This is because, under the assumption on $K$, the equilibrium measure $\mu_{K}$ is given by $\phi * d \theta$, that is,

$$
\int f d \mu_{K}=\frac{1}{2 \pi} \int_{0}^{2 \pi} f\left(\phi\left(e^{i \theta}\right)\right) d \theta \text {. }
$$

Since, $\mu_{n}=\frac{1}{n+1} \sum_{i=0}^{n}\left[a_{i}\right] \stackrel{\star}{\rightarrow} d \theta$, we have $\phi * \mu_{n}=\frac{1}{n+1} \sum_{i=0}^{n}\left[\phi\left(a_{i}\right)\right] \stackrel{\star}{\rightarrow} \phi * d \theta$ which implies that $\left(\phi\left(a_{n}\right)\right)$ is extremal. There is a short approximation theoretic argument that shows the relation $\mu_{K}=\phi * d \theta$. It is well known, see e.g. [16], that under the assumption on $K$, the Lagrange interpolation polynomials of any analytic function on a neighborhood of $K$ at the Fejer points

$$
\phi(\exp (2 i j \pi /(d+1))), \quad j=0, \ldots, d, d \in \mathbb{N},
$$

converge to $f$ uniformly on $K$. Since the points are located on the boundary of $K$, this implies that

$$
v_{d}=\frac{1}{d+1} \sum_{i=0}^{d}[\phi(\exp (2 i j \pi /(d+1)))] \stackrel{\star}{\longrightarrow} \mu_{K}
$$


Since, $v_{d}=\phi * \tau_{d}$ where

$$
\tau_{d}=\frac{1}{d+1} \sum_{i=0}^{d}[\exp (2 i j \pi /(d+1))] \stackrel{\star}{\longrightarrow} d \theta,
$$

the claim follows.

A similar argument shows that if $K$ and $K^{\prime}$ are two nonpolar polynomially convex compact subsets of the plane and if

1. $\left(a_{n}\right)$ is a pseudo Leja sequence on $K$ and

2. $\phi$ is the conformal mapping of the exterior of $K$ to the exterior of $K^{\prime}$ extends to a homeomorphism from $\partial K$ onto $\partial K^{\prime}$

then $\phi\left(a_{n}\right)$ is an extremal sequence for $K^{\prime}$.

This construction provides many explicit examples of extremal sequences. However, since, as shown in 2.3 (C), an extremal sequence is not necessarily a pseudo Leja sequence, the question remains to find explicit pseudo Leja sequences. The next result provides such a result with a stronger assumption on $K$. We actually show that the image of a Leja sequence for the unit disk under some conformal mappings is a pseudo Leja sequence. Our result relies heavily on some classical works of Alper. The sequence $\left(\phi\left(e_{n}\right)\right)$ where $\left(e_{n}\right)$ is the Leja sequence defined in (23) and $\phi$ is a conformal mapping was first studied and applied to the resolution of complex linear systems by iterative methods by Fischer and Reichel ([15], [14]). Let us finally mention that if the boundary of $K$ is sufficiently regular, Fejer points that we mentioned above are known to be close, in a certain sense, to Fekete points for $K$, see [21] and [22]. Finally, we mention that the Lebesgue constant of Leja sequences for the disk and of their images by conformal mappings are studied in [11].

\subsection{Pseudo Leja sequences on some Jordan curves}

Let $\Gamma$ be a smooth Jordan curve. We denote by $\theta(s)$ the angle between the tangent at $\Gamma(s)$ and the positive real axis where $s$ is the arc length parameter, thus $\theta(s)=\arg \left(\Gamma^{\prime}(s)\right)$. One says that $\Gamma$ is Alper-smooth if the modulus of continuity $\omega$ of $\theta$ satisfies

$$
\int_{0}^{h} \frac{\omega(x)}{x}|\log x| \mathrm{d} x<\infty .
$$

This notion was introduced in [1] and further used in [2] which we use later, see also [17]. Twice continuously differentiable Jordan curves are Alper-smooth. Indeed, in that case, the function $\theta(s)$ is continuously differentiable so that $\omega(x)=O(x)$ and the condition is satisfied.

Lemma 1 If $r_{0}, \ldots, r_{n-1}$ is a complete set of $n$-th roots of unity and $h$ is a continuous function of total variation $V<\infty$ on the unit circle, then

$$
\left|\frac{1}{2 \pi} \int_{0}^{2 \pi} h\left(e^{i t}\right) \mathrm{d} t-\frac{1}{n} \sum_{j=0}^{n-1} h\left(r_{j}\right)\right| \leq \frac{V}{n} .
$$

Proof Here, the order of the points does not matter and we may freely assume that $r_{j}=$ : $\exp (2 i j \pi / n)$. Since, moreover, the total variation $V$ is the sum of the variation $V_{j}$ on the intervals $[2 j \pi / n, 2(j+1) \pi / n], j=0, \ldots, n-1$, the rough estimate $V_{j} \geq\left|h\left(e^{i t}\right)-h\left(e^{2 i j \pi / n}\right)\right|$ yields the classical estimate of the lemma. 
Lemma 2 Let $\left(a_{n}\right)$ be a Leja sequence for the unit disk with $a_{0}=1$, and let $h$ be a continuous function of total variation $V<\infty$ on the unit circle. If $n=\sum_{j=0}^{k} \epsilon_{j} 2^{j}$ with $\epsilon_{j} \in\{0,1\}$ for $j=0, \ldots, k-1$ and $\epsilon_{k}=1$, then we have

$$
\left|\int h d \mu_{n-1}-\frac{1}{2 \pi} \int_{0}^{2 \pi} h\left(e^{i t}\right) d t\right| \leq \frac{V}{n} \sum_{j=0}^{k} \epsilon_{j},
$$

where $\mu_{n-1}=\frac{1}{n} \sum_{l=0}^{n-1}\left[a_{l}\right]$.

Proof The result is true for $n=1$ as follows from the previous lemma. We assume that the result is true for every $n \leq 2^{k}$ and prove it for every $m$ between $2^{k}+1$ and $2^{k+1}$. If $m=2^{k+1}$, then, in view of Theorem $5,\left\{a_{0}, \ldots, a_{m-1}\right\}$ forms a complete set of $2^{k+1}$-th roots of unity and lemma 1 gives the result. Now, if $2^{k}+1 \leq m \leq 2^{k+1}-1$, then

$$
m=\sum_{j=0}^{k-1} \epsilon_{j} 2^{j}+2^{k}, \quad \epsilon_{j} \in\{0,1\} .
$$

Now,

$$
\begin{aligned}
& \left|\frac{1}{m} \sum_{l=0}^{m-1} h\left(a_{l}\right)-\frac{1}{2 \pi} \int_{0}^{2 \pi} h\left(e^{i t}\right) \mathrm{d} t\right| \\
& \leq \frac{2^{k}}{m}\left|\frac{1}{2^{k}} \sum_{l=0}^{2^{k}-1} h\left(a_{l}\right)-\frac{1}{2 \pi} \int_{0}^{2 \pi} h\left(e^{i t}\right) \mathrm{d} t\right|+\left|\frac{1}{m} \sum_{l=2^{k}}^{m-1} h\left(a_{l}\right)-\frac{m-2^{k}}{m} \frac{1}{2 \pi} \int_{0}^{2 \pi} h\left(e^{i t}\right) \mathrm{d} t\right| \\
& \leq \frac{V}{m}+\frac{m-2^{k}}{m}\left|\frac{1}{m-2^{k}} \sum_{l=2^{k}}^{m-1} h\left(a_{l}\right)-\frac{1}{2 \pi} \int_{0}^{2 \pi} h\left(e^{i t}\right) \mathrm{d} t\right|
\end{aligned}
$$

where the last inequality is again a consequence of lemma 1 . We now concentrate on the right-hand term. In view of Theorem 5, we have

$$
\left(a_{2^{k}}, \ldots, a_{m}, \ldots, a_{2^{k+1}-1}\right)=\rho\left(b_{0}, \ldots, b_{m-2^{k}}, \ldots, b_{2^{k}-1}\right),
$$

where the $b_{i}$ 's are the $2^{k}$ first terms of a Leja sequence starting from 1 and $\rho=a_{2^{k}}$ satisfies $\rho^{2^{k}}=-1$. The function $h_{\rho}: z \rightarrow h(\rho z)$ has same integral and same total variation as $h$ on the unit disk. Moreover, we have $m-2^{k} \leq 2^{k}$. Hence, we may apply the induction hypothesis with $h_{\rho}$ and the Leja sequence $\left(b_{n}\right)$ to get

$$
\left|\frac{1}{m-2^{k}} \sum_{l=2^{k}}^{m-1} h\left(a_{l}\right)-\frac{1}{2 \pi} \int_{0}^{2 \pi} h\left(e^{i t}\right) \mathrm{d} t\right|=\left|\frac{1}{m-2^{k}} \sum_{l=0}^{m-1-2^{k}} h_{\rho}\left(b_{l}\right)-\frac{1}{2 \pi} \int_{0}^{2 \pi} h_{\rho}\left(e^{i t}\right) \mathrm{d} t\right| \leq \frac{V}{m-2^{k}} \sum_{j=0}^{k-1} \epsilon_{j} .
$$

The relations (39) and (40) now imply

$$
\left|\int h d \mu_{m-1}-\frac{1}{2 \pi} \int_{0}^{2 \pi} h\left(e^{i t}\right) \mathrm{d} t\right| \leq \frac{V}{m}\left(1+\sum_{j=0}^{k-1} \epsilon_{j}\right),
$$

which is the claimed relation for $m$ and this finishes the proof of the lemma. 
Theorem 6 Let $K$ be a compact set whose boundary is an Alper-smooth Jordan curve. Let $\phi$ be the conformal mapping of the exterior of the unit disk on the exterior of $K$. If $\left(a_{n}\right)$ is a Leja sequence for the unit disk, then $\left(\phi\left(a_{n}\right)\right)$ is a pseudo Leja sequence for $K$ of Edrei growth $c_{n}^{2}$ where

$$
c_{n}=\exp \left(A \sum_{j=0}^{k} \epsilon_{j}\right), \quad n=\sum_{j=0}^{k} \epsilon_{j} 2^{j}, \quad \epsilon_{j} \in\{0,1\},
$$

and $A$ is a positive constant depending only on $K$.

Observe that since $\sum_{j=0}^{k} \epsilon_{j} \leq \log _{2}(n+1)$, we have $c_{n} \leq(n+1)^{A / \log 2}$.

Lemma 3 Under the assumptions of Theorem 6, for any $w$ on the unit circle and $n \in \mathbb{N}^{*}$, we have

$$
C(K)^{n} \frac{1}{c_{n}} \leq \prod_{l=0}^{n-1} \frac{\left|\phi(w)-\phi\left(a_{l}\right)\right|}{\left|w-a_{l}\right|} \leq C(K)^{n} c_{n} .
$$

Proof The proof is an adaptation of the reasoning used on pp. 48-49 of [2]. Fix $w$ with $|w|=1$. Put

$$
f(z):=\log \left|\frac{\phi(w)-\phi(z)}{w-z}\right|, \quad|z| \geq 1 .
$$

We first examine $\int_{0}^{2 \pi} f\left(e^{i t}\right) \mathrm{d} t$. Observe that $f$ is harmonic in $\overline{\mathbb{C}} \backslash D(0,1)$ and continuous in $\mathbb{C} \backslash$ Int $D(0,1)$, because $\phi^{\prime}$ is continuous and does not vanish on $\mathbb{C} \backslash$ Int $D(0,1)$, see [1]. By the mean value theorem for harmonic functions,

$$
\frac{1}{2 \pi} \int_{0}^{2 \pi} f\left(e^{i t}\right) \mathrm{d} t=\lim _{z \rightarrow \infty} f(z) .
$$

Since $\phi(z)=c z+c_{0}+c_{1} z^{-1}+c_{2} z^{-2}+\cdots$ with $|c|=C(K)$ (see e.g. [23, Th.9.9]), we have $\lim _{z \rightarrow \infty} f(z) \log C(K)$ and thus

$$
\frac{1}{2 \pi} \int_{0}^{2 \pi} f\left(e^{i t}\right) \mathrm{d} t=\log C(K) .
$$

Our next claim is that $t \mapsto f\left(e^{i t}\right)$ is a function of bounded variation. It is proved in [1] that if the boundary of $K$ is an Alper-smooth Jordan curve, then there exists a constant $A$ independent of $w$ such that

$$
\int_{0}^{2 \pi}\left|\frac{\phi^{\prime}\left(e^{i \theta}\right)}{\phi\left(e^{i \theta}\right)-\phi(w)}-\frac{1}{e^{i \theta}-w}\right| \mathrm{d} \theta<A .
$$

On the other hand, we have

$$
\frac{\mathrm{d}}{\mathrm{d} t} \log \frac{\phi(w)-\phi\left(e^{i t}\right)}{w-e^{i t}}=i e^{i t}\left[\frac{\phi^{\prime}\left(e^{i t}\right)}{\phi\left(e^{i t}\right)-\phi(w)}-\frac{1}{e^{i t}-w}\right] .
$$


Consequently, $\log \frac{\phi(w)-\phi\left(e^{i t}\right)}{w-e^{i t}}$ is a function of total variation bounded by $A$ (as a function of $t \in[0,2 \pi))$. Therefore, so is its real part $f\left(e^{i t}\right)$. Lemma 2 and (43) now give

$$
\left|\frac{1}{n} \sum_{l=0}^{n-1} f\left(a_{l}\right)-\log C(K)\right| \leq \frac{\log c_{n}}{n} .
$$

Hence,

$$
c_{n}^{-1 / n} \leq \frac{1}{C(K)} \prod_{l=0}^{n-1}\left|\frac{\phi(w)-\phi\left(a_{l}\right)}{w-a_{l}}\right|^{1 / n} \leq c_{n}^{1 / n},
$$

which implies inequality (41).

Proof (Proof of Theorem 6) Using first the upper bound, then the lower bound given by lemma 3, we obtain for $n \geq 0$,

$$
\begin{aligned}
\prod_{j=0}^{n}\left|\phi\left(a_{n+1}\right)-\phi\left(a_{j}\right)\right| & \geq c_{n}^{-1} C^{n}(K) \prod_{j=0}^{n}\left|a_{n+1}-a_{j}\right| \\
& =c_{n}^{-1} C^{n}(K) \max _{|z|=1} \prod_{j=0}^{n}\left|z-a_{j}\right| \\
& \geq \frac{c_{n}^{-1} C^{n}(K)}{c_{n} C^{n}(K)} \max _{|z|=1} \prod_{j=0}^{n}\left|\phi(z)-\phi\left(a_{j}\right)\right| \\
& \geq c_{n}^{-2} \max _{w \in \Gamma} \prod_{j=0}^{n}\left|w-\phi\left(a_{j}\right)\right| .
\end{aligned}
$$

We used the fact that $\left(a_{n}\right)$ is a Leja sequence for the unit disk at the second line.

\section{Computing pseudo Leja sequences}

4.1 Weakly admissible meshes

We say that a sequence of sets $A_{n}, n \in \mathbb{N}$, is a weakly admissible mesh for $K$ if the following two conditions are satisfied.

1. $A_{n}$ is a finite subset of $K$.

2. There exists a sequence $\left(M_{n}\right)$ of subexponential growth (as $\left.n \rightarrow \infty\right)$ such that, for every polynomial $p$ of degree at most $n$,

$$
\max _{z \in K}|p(z)| \leq M_{n} \max _{z \in A_{n}}|p(z)|,
$$

The sequence $\left(M_{n}\right)$ is referred to as the growth of the mesh $\left(A_{n}\right)$. Such meshes were recently introduced in [10] (with the supplementary requirement that the cardinality of $A_{n}$ is also of subexponential growth as $n \rightarrow \infty)$. In the case where the sequence $M_{n}$ is bounded by $M$, we say that $\left(A_{n}\right)$ is an admissible mesh of parameter $M$. For another recent use of weakly admissible meshes in multivariate polynomial approximation, we refer to [8]. 
Proposition 1 Let $\left(A_{n}\right)$ be a weakly admissible mesh of growth $\left(M_{n}\right)$. We define inductively a sequence $\left(a_{n}\right)$ as follows. We take $a_{0} \in K$, and for $d \geq 1$, we select $a_{d} \in A_{d}$ such that

$$
\left|w_{d}\left(a_{d}\right)\right|=\max _{z \in A_{d}}\left|w_{d}(z)\right|
$$

where, as usual, $w_{d}(z)=\left(z-a_{0}\right) \cdots\left(z-a_{d-1}\right)$. Then, the sequence $\left(a_{n}\right)$ is a pseudo Leja sequence of Edrei growth $\left(M_{n}\right)$ for $K$.

Proof It suffices to observe that since $\operatorname{deg} w_{d}=d$, in view of (52), we have

$$
\max _{z \in K}\left|w_{d}(z)\right| \leq M_{d} \max _{z \in A_{d}}\left|w_{d}(z)\right|=M_{d}\left|w_{d}\left(a_{d}\right)\right| .
$$

This proposition reduces the problem of computing a pseudo Leja sequence for $K$ to that of constructing a weakly admissible mesh. The algorithm consists merely in computing $w_{d}\left(A_{d}\right)$ and selecting an element of $A_{d}$ for which the maximum is attained. To avoid an excessive computational cost, it is clearly desirable to keep the cardinality of $A_{n}$ as small as possible. In the following paragraph, we show how to construct weakly admissible meshes with few elements for a restricted but important class of compact sets including all polygons. In the next one, we show how to construct admissible meshes for a very general class of compact sets. The cost is much more expensive but still acceptable, at least if we require a reasonable number of points. Let us observe that in practice, it rapidly becomes impossible to compare the values in $\left|w_{d}\right|\left(A_{d}\right)$, simply because the values of the factors are too close to 0 . An easy way out is to compute $\log \left|w_{d}\right|\left(A_{d}\right)$ rather than $\left|w_{d}\right|\left(A_{d}\right)$.

4.2 Constructing weakly admissible meshes

First, recall that the Lebesgue constant $\Delta_{d}$ of a set of $d+1$ distinct interpolation points $A_{d}=\left\{z_{0}, \ldots, z_{d}\right\}$ in a compact subset $K$ is given by

$$
\Delta_{d}=\max _{z \in K} \sum_{j=0}^{d} \prod_{l \neq j}^{d}\left|\frac{z-z_{l}}{z_{j}-z_{l}}\right| .
$$

From the Lagrange interpolation formula, we have, for every polynomial $p$ of degree $d$,

$$
\max _{z \in K}|p(z)| \leq \Delta_{d} \max _{z \in A_{d}}|p(z)| .
$$

Thus, if, for every $d \geq 0$, we know a set of interpolation points $A_{d}$ with small Lebesgue constant, then $\left(A_{d}\right)$ will provide a weakly admissible mesh for $K$.

Here is a list of compact sets for which we know a set of interpolation points $A_{d}$ whose Lebesgue constant grows minimally, that is, like $\log d$.

1. A segment $[a, b]$ where $a$ and $b$ are two distinct complex numbers. Take $A_{d}=\phi\left(T_{d}^{-1}(0)\right)$ where $T_{d}^{-1}(0)$ denotes the roots of the $d$-th Chebyshev polynomial and $\phi$ is the affine map that sends $[-1,1]$ onto $[a, b]$.

2. A circle $C(a, r)$ (or a closed disk $\bar{D}(a, r))$. Take $A_{d}=\{\exp (2 i k \pi /(d+1)): 0 \leq k \leq d\}$.

3. More generally, a compact set bounded by an Alper-smooth Jordan curve,

$$
A_{d}=\{\phi(\exp (2 i k \pi /(d+1))): 0 \leq k \leq d\},
$$

see [2], where $\phi$ is as in theorem 6 . 
Actually, weakly admissible meshes can be readily constructed for any compact which is a (not necessarily disjoint) union of compact sets of the above types. Indeed, this follows from the fact that if $\left(A_{d, j}\right)$ is a weakly admissible mesh for $K_{j}, j=1, \ldots, s$, then $\left(\cup_{j=1}^{s} A_{d, j}\right)$ is a weakly admissible mesh for $K=\cup_{i=1}^{s} K_{j}$. Thus, for instance, if $K$ is a polygon with $s$ edges, we may construct an admissible mesh $\left(A_{d}\right)$ on $K$ with $M_{d}=O(\log d)$, and $A_{d}$ contains $s(d+1)$ points. This idea is illustrated in the examples given in $4.4(\mathrm{~A})$ below.

4.3 Constructing admissible meshes on a general compact set

We now explain how to construct an admissible mesh for a very general compact set $K$. We follow the construction given in [10] paying more attention to the parameters of the mesh. We need to use a precise Markov inequality. We refer to Sect. 2.4 for the notation.

Theorem 7 (Pommerenke [20]) Every compact connected subset $K$ in the complex plane is a $(e /(2 C(K)), 2)$ compact set.

Many important compact sets do have a Markov inequality of exponent smaller than 2. However, as far as we know, most authors concentrate on the exponent and a corresponding constant $\delta$ in general is difficult to estimate. In fact, Pommerenke's result is particularly useful for it requires very little information about $E$. Since, for a connected compact set, $c(E) \geq \operatorname{diam}(E) / 4$ ([30, corollary 5, p. 85]), it suffices to know a lower bound on the diameter of $E$. Thus, in practice, the parameters $(\delta, r)$ of the following lemma will be often taken as $(e /(2 D(E)), 2)$ where $D(E)$ is a (lower) estimate of the diameter of $E$.

Proposition 2 Let $M>1$ and let $K$ be a $(\delta, r)$ polynomially compact set whose boundary is given by a $C^{1}$-curve $\gamma:[a, b] \rightarrow \partial K$ with

$$
\max _{t \in[a, b]}\left|\gamma^{\prime}(t)\right| \leq D
$$

Then, the sets

$$
A_{n}=\left\{\gamma(a+k(b-a) / N): k=0, \ldots N, \quad N=\left\lceil 1 / f_{n}\right\rceil\right\}, \quad n \in \mathbb{N}^{\star},
$$

where

$$
f_{n}=\frac{2(M-1)}{(b-a) M D \delta n^{r}}
$$

form an admissible mesh of parameter $M$ for $K$. Here, $\left\lceil 1 / f_{n}\right\rceil$ denotes the smallest integer greater than $1 / f_{n}$.

As described earlier, since the union of admissible meshes $A_{n}^{j}$ of parameter $M$ for compacts $K_{j}, j=1, \ldots, s$, is itself an admissible mesh of parameter $M$ for $\cup_{j=1}^{r} K_{j}$, we may construct admissible meshes for any compact sets which is a finite union of compacts sets satisfying the conditions of Proposition 2.

Proof We must prove that if $p$ is a polynomial of degree $\leq n$, then

$$
\max _{z \in K}|p(z)| \leq M \max _{z \in A_{n}}|p(z)| .
$$

Let $z \in \partial K$ and $t \in[a, b]$ with $z=\gamma(t)$. By definition of $f_{n}$, there exists $k \in\{0, \ldots, N\}$ such that $\left|t-t_{k}\right| \leq(b-a) / 2 N$ with $t_{k}=a+k(b-a) / N$. Using the mean value theorem 
together with Markov's inequality, we obtain

$$
\begin{aligned}
\left|p(\gamma(t))-p\left(\gamma\left(t_{k}\right)\right)\right| & \leq\left|t-t_{k}\right| \max _{u \in[a, b]}\left|(p \circ \gamma)^{\prime}(u)\right| \\
& \leq\left|t-t_{k}\right| D \max _{z \in K}\left|p^{\prime}(z)\right| \\
& \leq \frac{b-a}{2 N} D \delta n^{r} \max _{z \in K}|p(z)| .
\end{aligned}
$$

By definition of $N$, we have $N=\left\lceil 1 / f_{n}\right\rceil$ hence $1 / N \leq f_{n}$. Now, in view of (57), Eq. (60) yields

$$
\left|p(\gamma(t))-p\left(\gamma\left(t_{k}\right)\right)\right| \leq \frac{M-1}{M} \max _{z \in K}|p(z)|
$$

It follows that

$$
|p(z)| \leq\left|p\left(\gamma\left(t_{k}\right)\right)\right|+(M-1) / M \max _{z \in K}|p(z)| \leq \max _{z \in A_{n}}|p(z)|+(M-1) / M \max _{z \in K}|p(z)| .
$$

Hence,

$$
\max _{z \in \partial K}|p(z)| \leq \max _{z \in A_{n}}|p(z)|+(M-1) / M \max _{z \in K}|p(z)| \Longrightarrow \max _{z \in K}|p(z)| \leq M \max _{z \in A_{n}}|p(z)| .
$$

There would be other definitions of $A_{n}$ based on the same idea. For example, we could take

$$
A_{n}=\left\{\gamma\left(a+k f_{n}(b-a)\right): k=0, \ldots,\left\lfloor 1 / f_{n}\right\rfloor\right\} \cup\{\gamma(b)\} .
$$

Also, of course, different parametrizations of the boundary of $K$ would provide other admissible meshes.

Observe finally that in the case where $K$ is an interval $[a, b](\gamma: t \in[a, b] \rightarrow t)$, then $(\delta, r)=(2 /(b-a), 2), D=1$ and the set $A_{n}$ is formed of equidistantly distributed points, namely

$$
A_{n}=\left\{a+k(b-a) / N: k=0, \ldots N, N=\left\lceil(M-1) n^{2}\right\rceil\right\} .
$$

These meshes are used in 4.4 (D) below.

\subsection{Examples}

(A) In fig. 1 (I), we show the first points of a pseudo Leja sequence on the union $K_{1}$ of the two intervals of different lengths $[-10,-6]$ and $[5,8]$. We actually show $a_{j}+i j / 10$ so that the $y$-coordinate gives the position of the point within the sequence whereas the $x$-coordinate is the actual value of the point.

The computation is based on proposition 1 . We used the weakly admissible mesh of Chebyshev type formed by

$$
A_{n}\left(K_{1}\right)=\bigcup_{i=1,2}\left\{\frac{l_{i}-r_{i}}{2} \cos \left(\frac{2 j+1}{n+1} \frac{\pi}{2}\right)+\left(l_{i}+r_{i}\right) / 2: j=0, \ldots, n\right\},
$$

where $\left[l_{1}, r_{1}\right]=[-10,-6]$ and $\left[l_{2}, r_{2}\right]=[5,8]$. The interval of greater length must contain more points. This is illustrated by the fact that on rare occasions (here twice), two successive points of our pseudo Leja sequence are selected within the same interval. 


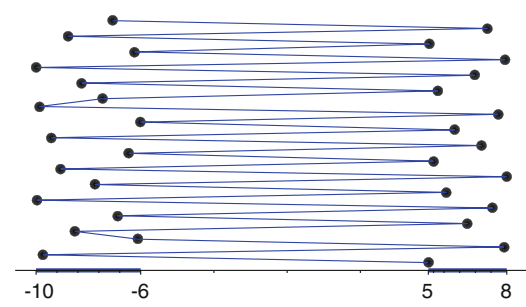

(I) First 32 points of a pseudo Leja sequence for a union of intervals of different lengths : $K_{1}=$ $[-10,-6] \cup[5,8]$

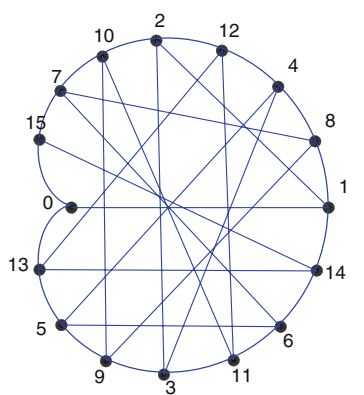

(II) First 16 points of a pseudo Leja sequence of Edrei coefficient 4 on the cardioid $\left\{\left(x^{2}+y^{2}-\right.\right.$ $\left.x)^{2}=x^{2}+y^{2}\right\}, z=x+i y$.

Fig. 1 Examples of Pseudo Leja points for a union of intervals of different lengths and for a regular curve

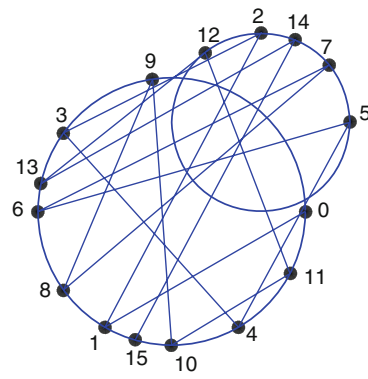

$$
K_{2}=D(0,3 / 2) \cup D(1+i, 1)
$$

(I) 32 points

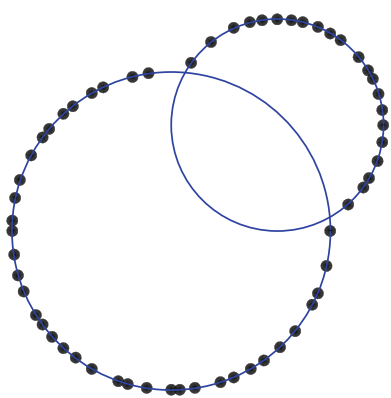

$K_{2}=D(0,3 / 2) \cup D(1+i, 1)$

(II) 60 points

Fig. 2 First points of a pseudo Leja sequence for two overlapping disks

(B) The points on the cardioid in Fig. 1 (II) are computed with the help of proposition 2. The cardioid is parametrized by

$$
\gamma: t \in[0,2 \pi] \rightarrow \frac{1}{2}(1+2 \exp (i t)+\exp (2 i t)) .
$$

Here, the parameters needed to use proposition 2 are $D=2$ and

$$
(\delta, r)=(e /(2 C), 2), \quad C=1 \leq C(\kappa),
$$

the later inequality coming from the fact that the disk of center $a=1$ and radius $\rho=1$ lies within $\kappa$. Numerical experiments show that points are denser on the opposite side of the cusp and near, but away, on both sides of the cups.

(C) In Fig. 2, we show pseudo Leja points on the union $K_{2}$ of the two overlapping disks $D(0,3 / 2)$ and $D(1+i, 1)$ using again proposition 1 with

$$
A_{n}\left(K_{2}\right)=\bigcup_{i=1,2}\left\{c_{i}+\rho_{i} \exp (2 i k \pi /(n+1)): k=0, \ldots, n\right\},
$$

where $D\left(c_{1}, \rho_{1}\right)=D(0,3 / 2)$ and $D\left(c_{2}, \rho_{2}\right)=D(1+i, 1)$. The weakly admissible mesh $A_{n}\left(K_{2}\right)$ contains many points in the interior of $K_{2}$. The theory tells us that a pseudo 
Table 1 Error in the approximation of a Runge-type function by interpolation at various pseudo Leja points

\begin{tabular}{lllllll}
\hline Edrei growth & \multicolumn{5}{c}{ Number of interpolation points } \\
\cline { 2 - 6 }$M$ or $M_{n}$ & $N=10$ & $N=50$ & $N=100$ & $N=150$ & $N=200$ & $N=250$ \\
\hline$M=1.05$ & 0.51 & $2.2 \times 10^{-2}$ & $2.38 \times 10^{-4}$ & $4.3 \times 10^{-6}$ & $1.05 \times 10^{-8}$ & $7.28 \times 10^{-11}$ \\
$M=1.5$ & 0.51 & $2.8 \times 10^{-2}$ & $2.12 \times 10^{-4}$ & $5 \times 10^{-7}$ & $1.4 \times 10^{-8}$ & $9 \times 10^{-11}$ \\
$M_{n}=O(\log n)$ & 0.67 & $1.8 \times 10^{-2}$ & $1.6 \times 10^{-4}$ & $1.3 \times 10^{-6}$ & $1.21 \times 10^{-8}$ & $1.06 \times 10^{-10}$ \\
\hline
\end{tabular}

Leja sequence can only have a small number of points in the interior of the compact set but the algorithm actually selected no such point.

(D) Table 1 shows the error between the Runge-type function $f$ defined on $[-1,1]$ by $f(t)=\left(1+100 t^{2}\right)^{-1}$ and its interpolation polynomials $\mathbf{L}\left[a_{0}, \ldots, a_{N-1} ; f\right]$ computed at various kinds of pseudo Leja points for the interval $K=[-1,1]$. The singularities of $f, z= \pm i / 10$, are very close to $[-1,1]$, and this implies a relatively slow convergence of the best approximation polynomials. Actually, since the singularities lie on the ellipse $K_{R}=\left\{(R / 2) \exp (i \theta)+\left(R^{-1} / 2\right) \exp (-i \theta): \theta \in[0,2 \pi]\right\}$, see (4), with $R=(\sqrt{101}+1) / 10 \approx 1.105$, the Bernstein-Walsh theorem [31, §4.7] says that the uniform distance between $f$ and the space of polynomials of degree at most $n$ decreases approximately like $(1 / R)^{n}$. We estimated the uniform norm $\max _{t \in[-1,1]}\left|f(t)-\mathbf{L}\left[a_{0}, \ldots, a_{N-1} ; f\right](t)\right|$ by computing the error on a large discrete subset of $[-1,1]\left(10^{4}\right.$ equispaced points). The results suggest that the value of the Edrei coefficient (growth) plays a limited role even in the distribution of the first points. This is in accordance with the estimates given in subsection 2.4. In the particular case of the function $f$ above, the best Edrei growth provides a better approximation only for a very small number of interpolation points. On the other hand, the choices $M=1.01$ and $M=1.5$ induce a much higher computational cost. Observe that, as expected (see Theorem 1 and its second consequence), in every case, we obtain a nearly optimal precision, that is, close to $(1 / R)^{(n-1)}$ where $n$ is the number of points (for instance, for $\left.n=250,(1 / R)^{(n-1)} \approx 1.610^{-11}\right)$.

\section{Multivariate Lagrange interpolation}

Let

$$
A_{i}=\left\{a_{i j}, \quad 0 \leq j \leq d\right\} \subset \mathbb{C}, \quad i=1, \ldots, N,
$$

where the $d+1$ points $a_{i j}$ are pairwise distinct. We define a subset $A$ of $\mathbb{C}^{N}$ depending on $\left(A_{1}, \ldots, A_{n}\right)$ by setting

$$
A=\left\{a_{\alpha}:=\left(a_{1, \alpha_{1}}, a_{2, \alpha_{2}}, \ldots, a_{N, \alpha_{N}}\right), \quad|\alpha| \leq d\right\},
$$

where $|\alpha|=\alpha_{1}+\cdots+\alpha_{N}$ denotes the length of the multi-index $\alpha=\left(\alpha_{1}, \ldots, \alpha_{N}\right)$. It is not difficult to see that $A$ contains $\left(\begin{array}{c}N+d \\ d\end{array}\right)$ points which is the dimension of the space of polynomials of degree at most $d$ on $\mathbb{C}^{N}$. A classical result of Biermann established in 1903 [5] asserts that $A$ is unisolvent for Lagrange interpolation of degree $d$. This means that, given any function $f$ defined on $A$, there exists one and only one polynomial $p$ of degree at 
most $d$ in $N$ complex variables such that $p=f$ on $A$. This polynomial $p$ is the multivariate Lagrange interpolation polynomial of $f$ at the points of $A$. We denote it by $\mathbf{L}[A ; f]$. We write

$$
A=A_{1} \otimes A_{2} \otimes \cdots \otimes A_{N}
$$

and call $A$ the Biermann product of $A_{1}, \ldots, A_{N}$. References on Biermann products can be found in [9] where a generalization is studied.

The main application of our pseudo Leja sequences stems from the following theorem which shows that they enable one to construct extremal sets for multivariate Lagrange interpolation of analytic functions. The use of Corollary 3 or Theorem 6 actually provides explicit theoretical examples while the method given in Sect. 4 gives a way of computing such points in rather general cases, see the examples below. The classical case of Leja sequences was first established by Siciak [27]. The proof extends to the case of our pseudo Leja sequences. We shall sketch it for the convenience of the reader and to emphasize the importance of working here with sequences rather than with arrays.

Theorem 8 Let $K_{i}$ be a regular polynomially convex compact set in the complex plane, $i=1, \ldots, N$, and $a_{i}=\left(a_{i, n}\right)$ a pseudo Leja sequence for $K_{i}$ or, more generally a sequence for which

$$
\frac{1}{d+1} \sum_{j=0}^{d}\left[a_{i, j}\right] \stackrel{\star}{\rightarrow} \mu_{K_{i}} \quad d \rightarrow \infty
$$

We define

$$
A^{d}=\left\{a_{\alpha}=\left(a_{1, \alpha_{1}}, \ldots, a_{N, \alpha_{N}}\right):|\alpha| \leq d\right\} .
$$

For every function $f$ analytic on a neighborhood of

$$
K=K_{1} \times K_{2} \times \cdots \times K_{N} \subset \mathbb{C}^{N},
$$

we have

$$
\lim _{d \rightarrow \infty} \mathbf{L}\left[A^{d} ; f\right]=f, \text { uniformly on } K .
$$

The speed of convergence is actually geometric and maximal, that is, asymptotically equal to the speed provided by polynomials of best uniform approximation,

$$
\limsup _{d \rightarrow \infty}\left\{\max _{z \in K}\left|f(z)-\mathbf{L}\left[A^{d} ; f\right](z)\right|\right\}^{1 / d}=\limsup _{d \rightarrow \infty}\left\{\min _{\operatorname{deg} p=d} \max _{z \in K}|f(z)-p(z)|\right\}^{1 / d}<1 .
$$

This follows from a careful look at the proof below or from a general result on approximation by polynomial projectors, see [7, Theorem 7].

\subsection{Proof of theorem 8}

For sake of notational simplicity, we prove the result in the bivariate case only. Let $d \geq 0$ and let us write

$$
w_{\alpha}\left(z_{1}, z_{2}\right)=w_{1, \alpha_{1}}\left(z_{1}\right) \cdot w_{2, \alpha_{2}}\left(z_{2}\right), \quad \alpha=\left(\alpha_{1}, \alpha_{2}\right),
$$


where as usual

$$
w_{i \alpha_{i}}=\left(z-a_{i, 0}\right) \cdots\left(z-a_{i, \alpha_{i}-1}\right), \quad i=1,2 .
$$

Since the polynomials $w_{\alpha},|\alpha| \leq d$, form a basis of the space of polynomials of degree at most $d$, there exist coefficients $D_{\alpha}(f)$ such that

$$
\mathbf{L}\left[A^{d} ; f\right]\left(z_{1}, z_{2}\right)=\sum_{|\alpha| \leq d} D_{\alpha}(f) w_{\alpha}\left(z_{1}, z_{2}\right) .
$$

A priori, the $D_{\alpha}$ 's depend on $A^{d}$ and hence on $d$. However, since $A$ is constructed with sequences rather than with arrays, we have $A^{d_{1}} \subset A^{d_{2}}$ whenever $d_{1} \leq d_{2}$ and the coefficients $D_{\alpha}$ (and the polynomials $w_{\alpha}$ ) actually do not depend on $d$. This is the fundamental point. We state it carefully in the next lemma.

Lemma 4 If $|\alpha| \leq \min \left\{d_{1}, d_{2}\right\}$, then the coefficient $D_{\alpha}$ corresponding to $\mathbf{L}\left[A^{d_{1}} ; f\right]$ is equal to that corresponding to $\mathbf{L}\left[A^{d_{2}} ; f\right]$. Thus, we may speak of the series

$$
\sum_{|\alpha|=0}^{\infty} D_{\alpha}(f) w_{\alpha}
$$

and the convergence of the sequence of polynomials $\mathbf{L}\left[A^{d} ; f\right]$ is equivalent to the convergence of (65).

To establish the convergence of this series, we need an integral formula for the coefficients $D_{\alpha}(f)$, and to obtain this formula, we first establish an expression for the interpolating polynomial of a product function.

Lemma 5 If $f\left(z_{1}, z_{2}\right)=f_{1}\left(z_{1}\right) f_{2}\left(z_{2}\right)$, then

$$
\mathbf{L}\left[A^{d} ; f\right]=\sum_{|\alpha| \leq d} f_{1}\left[a_{10}, \ldots, a_{1 \alpha_{1}}\right] w_{\alpha_{1}}\left(z_{1}\right) \dot{f}_{2}\left[a_{20}, \ldots, a_{2 \alpha_{2}}\right] w_{\alpha_{2}}\left(z_{2}\right) .
$$

Proof Let us call the polynomial on the right-hand side, $p$. Since $p$ is a polynomial of degree $\leq d$, to prove $\mathbf{L}\left[A^{d} ; f\right]=p$, it suffices to check that $p=f$ on $A$. This follows from the fact that

$$
p\left(z_{1}, z_{2}\right)=\mathbf{L}\left[a_{10}, \ldots, a_{1 d}\right]\left(f_{1}\right)\left(z_{1}\right) \cdot \mathbf{L}\left[a_{20}, \ldots, a_{2 d}\right]\left(f_{2}\right)\left(z_{2}\right) \text { on } A .
$$

To see this, we just check that

$$
\sum_{|\alpha|>d} f_{1}\left[a_{10}, \ldots, a_{1 \alpha_{1}}\right] w_{\alpha_{1}}\left(z_{1}\right) \cdot f_{2}\left[a_{20}, \ldots, a_{2 \alpha_{2}}\right] w_{\alpha_{2}}\left(z_{2}\right)=0 \text { on } \mathrm{A},
$$

and this is because when $\alpha_{1}+\alpha_{2}>d$ and $i+j \leq d$ then $\alpha_{1}>i$ or $\alpha_{2}>j$ so that, for every $\alpha$, at least one of the two factors in each term of the sum (67) vanishes at $\left(a_{1 i}, a_{2 j}\right)$. For a more detailed treatment (in a general case), the reader may consult [9].

Now that (66) is established, comparison with (64) yields

$$
D_{\alpha}\left(f_{1} f_{2}\right)=f_{1}\left[a_{10}, \ldots, a_{1, \alpha_{1}}\right] \cdot f_{2}\left[a_{20}, \ldots, a_{2, \alpha_{2}}\right] .
$$

Now, applying the lemma to the Cauchy kernel and using a classical result on divided differences, we obtain

$$
D_{\alpha}\left(\frac{1}{v-\cdot 1} \frac{1}{w-\cdot 2}\right)=\frac{1}{\left(v-a_{1,0}\right) \cdots\left(v-a_{1, i}\right)} \cdot \frac{1}{\left(w-a_{2,0}\right) \cdots\left(w-a_{2, j}\right)} .
$$


The continuity of $D_{\alpha}$ as a function of $f$ and the bivariate Cauchy formula on the $K_{1 r} \times K_{2 r}$,

$$
f\left(z_{1}, z_{2}\right)=\frac{1}{(2 i \pi)^{2}} \int_{\partial K_{1 r}} \int_{\partial K_{2 r}} \frac{f(v, w) \mathrm{d} v \mathrm{~d} w}{\left(v-z_{1}\right)\left(w-z_{2}\right)}
$$

where $z_{1}\left(\right.$ resp. $\left.z_{2}\right)$ lies in the interior $K_{1 r}\left(\operatorname{resp} . K_{2 r}\right)$, give

$$
\begin{aligned}
D_{\alpha}(f) & =\frac{1}{(2 i \pi)^{2}} \int_{\partial K_{1 r}} \int_{\partial K_{2 r}} \mathrm{D}_{\alpha}\left(\frac{f(v, w)}{(v-\cdot 1)(w-\cdot 2)}\right) \mathrm{d} v \mathrm{~d} w \\
& =\frac{1}{(2 i \pi)^{2}} \int_{\partial K_{1 r}} \int_{\partial K_{2 r}} \frac{f(v, w) \mathrm{d} v \mathrm{~d} w}{\left(v-a_{1,0}\right) \cdots\left(v-a_{1, \alpha_{1}}\right)\left(w-a_{2,0}\right) \cdots\left(w-a_{2, \alpha_{2}}\right)}
\end{aligned}
$$

Here, we chose $r>1$ small enough to have $f$ analytic on a neighborhood of $K_{1 r} \times K_{2 r}$ where $K_{i r}$ denotes the level set of the Green function of $K_{i}$ as defined in (4). We denoted by $\partial K_{i r}$ the finite number of disjoints Jordan curves that form the boundary of $K_{i r}$.

Now, we take $\left(z_{1}, z_{2}\right)$ on $\partial K_{1 r^{\prime}} \times \partial K_{2 r^{\prime}}$ where $r^{\prime}<r$ will be fixed later and $\epsilon$ such that $r^{\prime}+\epsilon<r-\epsilon$. Since $\left|w_{i d}\left(z_{i}\right)\right|^{1 / d}$ converges to $G_{k_{i}}$ locally uniformly on $\mathbb{C} \backslash K_{i}$ (see the consequence (1) of Theorem 1), there exist constants $\Lambda$ and $\Lambda^{\prime}$ such that for $(v, w) \in \partial K_{1 r} \times \partial K_{2 r}$ and $\left(z_{1}, z_{2}\right) \in \partial K_{1 r^{\prime}} \times \partial K_{2 r^{\prime}}$, we have

$$
\frac{\left|\left(z_{1}-a_{1,0}\right) \cdots\left(z_{1}-a_{1, \alpha_{1}}\right)\left(z_{2}-a_{2,0}\right) \cdots\left(z_{2}-a_{2, \alpha_{2}}\right)\right|}{\left|\left(v-a_{1,0}\right) \cdots\left(v-a_{1, \alpha_{1}}\right)\left(w-a_{2,0}\right) \cdots\left(w-a_{2, \alpha_{2}}\right)\right|} \leq \frac{\Lambda^{\prime}}{\Lambda}\left(\frac{r^{\prime}+\epsilon}{r-\epsilon}\right)^{\alpha_{1}+\alpha_{2}} .
$$

Since $\left(r^{\prime}+\epsilon\right) /(r-\epsilon)<1$, the above estimate together with (71) and (64) shows that $\mathbf{L}\left[A^{d} ; f\right]$ converges uniformly on $\partial K_{1 r^{\prime}} \times \partial K_{2 r^{\prime}}$, hence also on $K_{1 r^{\prime}} \times K_{2 r^{\prime}}$, and its limit $F$ is therefore a function analytic on a neighborhood of $K$. Again, from (64), we see that for every fixed $\alpha, F\left(a_{\alpha}\right)=\mathbf{L}\left[A^{d} ; f\right]\left(a_{\alpha}\right)$ whenever $d \geq|\alpha|$. Hence, $F\left(a_{\alpha}\right)=f\left(a_{\alpha}\right)$, for every $\alpha$. Since the sets of all the points $a_{\alpha}$ is easily seen to be a set of uniqueness for functions analytic on a neighborhood of $K$, we must have $F=f$ and this completes the proof of the theorem.

Examples of multivariate interpolation points given by the method discussed here as well as further univariate illustrations and codes for computing pseudo Leja points are available from the authors.

Open Access This article is distributed under the terms of the Creative Commons Attribution Noncommercial License which permits any noncommercial use, distribution, and reproduction in any medium, provided the original author(s) and source are credited.

\section{References}

1. Al'per, S.Ya.: On uniform approximations of functions of a complex variable in a closed region. Izv. Akad. Nauk SSSR. Ser. Mat. 19, 423-444 (1955)

2. Al'per, S. Ya.: On the convergence of Lagrange's interpolational polynomials in the complex domain. Uspehi Mat. Nauk (N.S.) 11(5(71)), 44-50 (1956)

3. Baglama, J., Calvetti, D., Reichel, L.: Fast Leja points. Electron. Trans. Numer. Anal. 7:124-140 (1998) (electronic), Large scale eigenvalue problems (Argonne, IL, 1997)

4. Białas-Cież, L.: Markov sets in C are not polar. Bull. Polish Acad. Sci. Math. 46(1), 83-89 (1998)

5. Bierman, O.: Über näherungsweise kubaturen. Monaths. Math. Phys. 14, 211-225 (1903)

6. Bloom, T., Bos, L., Christensen, C., Levenberg, N.: Polynomial interpolation of holomorphic functions in $\mathbf{C}$ and $\mathbf{C}^{n}$. Rocky Mountain J. Math. 22(2), 441-470 (1992) 
7. Bloom, T., Calvi, J.-P.: The distribution of extremal points for Kergin interpolation: real case. Ann. Inst. Fourier (Grenoble) 48(1), 205-222 (1998)

8. Bos, L., Calvi, J.-P., Levenberg, N., Sommariva, A., Vianello, M.: Geometric weakly admissible meshes, discrete least squares approximations and approximate Fekete points. Preprint (2009), to appear in Math. Comp.

9. Calvi, J.-P.: Intertwining unisolvent arrays for multivariate Lagrange interpolation. Adv. Comput. Math. 23(4), 393-414 (2005)

10. Calvi, J.-P., Levenberg, N.: Uniform approximation by discrete least squares polynomials. J. Approx. Th. 152(1), 82-100 (2008)

11. Calvi, J.-P., Phung, Van, M.: On the Lebesgue constant of Leja sequences for the unit disk and its applications. Preprint (2010)

12. De Marchi, S.: On Leja sequences: some results and applications. Appl. Math. Comput. 152(3), 621647 (2004)

13. Edrei, A.: Sur les déterminants récurrents et les singularités d'une fonction donnée par son développement. Compositio Math. 7, 20-88 (1939)

14. Fischer, B., Reichel, L.: A stable Richardson iteration method for complex linear systems. Numer. Math. 54(2), 225-242 (1988)

15. Fischer, B., Reichel, L.: Newton interpolation in Fejér and Chebyshev points. Math. Comp. 53 (187), 265278 (1989)

16. Gaier, D.: Lectures on complex approximation. Birkhäuser Boston Inc., Boston, MA, 1987. Translated from the German by Renate McLaughlin

17. Kövari, T., Pommerenke, Ch.: On the distribution of Fekete points. Mathematika 15, 70-75 (1968)

18. Leja, F.: Sur certaines suites liées aux ensembles plans et leur application à la représentation conforme. Ann. Polon. Math. 4, 8-13 (1957)

19. Phung, Van M.: On the limit points of pseudo Leja sequences. Preprint (2010)

20. Pommerenke, Ch.: On the derivative of a polynomial. Michigan Math. J. 6, 373-375 (1959)

21. Pommerenke, Ch.: Über die Verteilung der Fekete-Punkte. Math. Ann. 168, 111-127 (1967)

22. Pommerenke, Ch.: Über die Verteilung der Fekete-Punkte. II. Math. Ann. 179, 212-218 (1969)

23. Pommerenke, Ch.: Boundary behaviour of conformal maps volume 299 of Grundlehren der Mathematischen Wissenschaften [Fundamental Principles of Mathematical Sciences]. Springer-Verlag, Berlin (1992)

24. Ransford, T.: Potential theory in the complex plane volume 28 of London Mathematical Society Student Texts. Cambridge University Press, Cambridge (1995)

25. Reichel, L.: Newton interpolation at Leja points. BIT 30(2), 332-346 (1990)

26. Saff, E.B., Totik, V: Logarithmic potentials with external fields, volume 316 of Grundlehren der Mathematischen Wissenschaften [Fundamental Principles of Mathematical Sciences]. Springer-Verlag, Berlin (1997). Appendix B by Thomas Bloom

27. Siciak, J.: On some extremal functions and their applications in the theory of analytic functions of several complex variables. Trans. Amer. Math. Soc. 105, 322-357 (1962)

28. Smirnov, V.I., Lebedev, N.A.: Functions of a complex variable: Constructive theory. Translated from the Russian by Scripta Technica Ltd. The M.I.T. Press, Cambridge (1968)

29. Taylor, R., Totik, V.: Lebesgue constants for Leja points. IMA J. Numer Anal. 30, 462-486 (2010)

30. Tsuji, M: Potential theory in modern function theory. Chelsea Publishing Co., New York (1975). Reprinting of the 1959 original

31. Walsh, J.L: Interpolation and approximation by rational functions in the complex domain. Fourth edition. American Mathematical Society Colloquium Publications, Vol XX. American Mathematical Society, Providence, RI (1965) 